\title{
Effects of liver function, insulin resistance and inflammatory factors on vascular endothelial dilation function and prognosis of coronary heart disease patients complicated with NAFLD
}

\author{
BIN WANG, FANG LI, JINGJING GUO, CHANGMEI WANG, DASHUAI XU and CONGCONG LI \\ Department of Geriatrics, Jinan Central Hospital, Shandong University, Jinan, Shandong 250013, P.R. China
}

Received September 7, 2017; Accepted November 23, 2018

DOI: $10.3892 /$ etm.2018.7043

\begin{abstract}
Effects of liver function, insulin resistance (IR) and inflammatory factors on vascular endothelial dilation function and prognosis of coronary heart disease (CHD) patients complicated with non-alcoholic fatty liver disease (NAFLD) were investigated. A total of 80 patients with CHD treated in Jinan Central Hospital from October 2016 to July 2017 were randomly enrolled and divided into the NAFLD group $(n=41)$ and the simple CHD group $(n=39)$. The IR, the vascular endothelial dilation function and the related inflammatory factors were also detected, followed by statistical analysis. The level of high-sensitivity C-reactive protein (hs-CRP), serum total bilirubin and alanine aminotransferase (ALT) levels and body mass index in the NAFLD group was decreased and the expression of tumor necrosis factor- $\alpha$ was increased compared with that in the simple CHD group $(\mathrm{P}<0.05)$. There was a linearly positive correlation between ALT and brachial artery diameter in the NAFLD group $(\mathrm{r}=0.311, \mathrm{P}<0.05)$. There was a correlation between homeostasis model assessment-IR (HOMA-IR) and HOMA- $\beta$ indexes and brachial artery diameter, hs-CRP and brachial artery diameter in both groups $(\mathrm{r}=-0.128, \mathrm{r}=0.219, \mathrm{P}<0.05)$. The HOMA indexes in the NAFLD group were increased compared with those in the simple CHD group $(\mathrm{P}<0.01)$. There were significant differences in the intima-media thickness, number of carotid plaques and detection rate of carotid plaques $(\mathrm{P}<0.05)$. The risk of cardiovascular events within 10 years in the NAFLD group was increased compared with that in the simple CHD group. The differences of incidence of cardiovascular diseases (CVD)s were statistically significant $(\mathrm{P}<0.05)$. Therefore, The changes in liver function indexes, IR and related inflammatory factors in CHD patients complicated with NAFLD significantly affect the vascular endothelial dilation function, which also have some effects on the occurrence of CVDs.
\end{abstract}

Correspondence to: Dr Changmei Wang, Department of Geriatrics, Jinan Central Hospital, Shandong University, 105 Jiefang Road, Jinan, Shandong 250013, P.R. China

E-mail: wangchangmeisd@126.com

Key words: nonalcoholic fatty liver disease, insulin resistance, coronary heart disease, inflammatory factors

\section{Introduction}

Non-alcoholic fatty liver disease (NAFLD) has been thought to be a benign disease, which is often associated with the central obesity, insulin resistance (IR) and metabolic syndrome. However, studies showed that $(1,2)$ NAFLD is a chronic disease, including benign lesions and significant liver damage, such as the lobular inflammation, ballooning degeneration of liver cells, liver fibrosis and cirrhosis (3). According to statistics, more than $30 \%$ of adults in the United States and other Western countries suffer from NAFLD (4), and the morbidity rate of those NAFLD patients with diabetes mellitus (DM) and transaminase is not included (5-7). NAFLD often occurs in patients with obesity and type 2 DM (T2DM), and its incidence rate is $57 \%$ in patients with obesity and $70 \%$ in patients with T2DM $(4,8)$. In addition to hepatic complications, the risk of cardiac metabolic complications, such as T2DM and cardiovascular disease (CVD), is also increased in NAFLD patients $(4,9)$. Coronary heart disease (CHD) is a common complication. The incidence of NAFLD combined with CHD is increasing gradually. It affects the digestive system and cardiovascular system including the liver, kidney and heart. The factors that cause CHD complicated NAFLD are various, influenced by external environment and diet habits, and are related to genetic factors of family (6). At present, there are few reports on the treatment of complications, mainly in the combination of diet control and cardiovascular drugs. The prognosis is poor, with easy relapse, and the treatment effect is not satisfactory (7). Endothelial dysfunction is an independent predictor of prognosis of CHD, including endothelium derived vasodilation, endothelium derived vasoconstrictor function generation, inflammatory and immune responses to reactive oxygen and nitrogen activation. In this study, the effects of liver function, IR and inflammatory factors on vascular endothelial dilation function and prognosis of CHD patients complicated with NAFLD were investigated.

\section{Patients and methods}

General materials. A total of 80 patients with CHD treated in Jinan Central Hospital (Jinan, China) from October 2016 to July 2017 were randomly enrolled, including 42 males and 38 females aged $52.5 \pm 8.8$ years on average. The diagnostic criteria of CHD: i) typical angina pectoris or suspected angina 
pectoris and oral nitroglycerin remission within $5 \mathrm{~min}$; ii) hypertensive history with left ventricular enlargement and aortic node calcification by X-ray, left ventricular high voltage by ECG; iii) patients with old myocardial infarction, myocardial infarction graphics by ECG, except those with suspected myocardial infarction graphics caused by other causes; iv) left axis deviation, left precordial lead with ST-T change by ECG; and v) left axis deviation is not more than $30^{\circ}$, with $\mathrm{A} 2>\mathrm{P} 2$ or apical systolic murmur, and it cannot be explained by other reasons. All patients were divided into the NAFLD group $(n=41)$, including 21 males and 20 females aged $49.6 \pm 8.3$ years on average, and the simple CHD group $(n=39)$, including 20 males and 19 females aged $50.2 \pm 9.0$ years on average according to whether they were complicated with NAFLD. There were no statistically significant differences in the sex and age between the two groups $(\mathrm{P}>0.05)$. The diagnosis of NAFLD met the diagnostic criteria in the Guidelines for the Diagnosis and Treatment of NAFLD (revised in 2010). The exclusion criteria i) standard: alcohol and other clear liver injury caused by serious liver disease, such as fatty liver, hepatitis, hepatocirrhosis, liver cancer, and liver ascites; ii) diabetics; iii) other organs with obvious inflammation; and iv) patients with mental disorders who cannot cooperate with the study. The study was approved by the Ethics Committee of Jinan Central Hospital and informed consents were signed by the patients or guardians.

\section{Research methods}

Determination of laboratory indexes. The height and weight of all subjects were measured, the body mass index (BMI) was calculated, and the venous blood was drawn to detect the fasting blood glucose (FBG), liver functions [alanine aminotransferase (ALT), aspartate aminotransferase (AST) and total bilirubin (TBIL)] and triglyceride (TG).

IR assessment. The fasting insulin (FINS) was detected using the full-automatic biochemical detector; IR was evaluated using the homeostasis model assessment-IR (HOMA-IR): HOMA-IR $=($ FINS x FBG $) / 22.5$; the islet $\beta$-cell basic function was expressed as the insulin secretion index (HOMA- $\beta$ ): HOMA- $\beta=20 \times$ FINS/(FBG-3.5).

Determination of vascular endothelial dilation function. LOGIQ-500 color ultrasound and 7.0 linear array probe (GE Healthcare, Chicago, IL, USA) with the detection depth of $4 \mathrm{~cm}$ was used to detect the brachial artery diameter under resting state after oral administration of nitroglycerin to reflect the blood flow changes caused by the vascular reactive hyperemia (vascular endothelium-dependent dilation function, FMV). The ultrasonic scanning of brachial artery was performed to measure the diameter of vessel in the three cases: i) in rest state, namely resting for at least $10 \mathrm{~min}$; ii) after the reactive hyperemia test (vascular compression and expansion caused by inflation and deflation of cuff of sphygmomanometer), namely after the cuff compression to $250 \mathrm{mmHg}$ or after discharge of cuff; and iii) after the sublingual administration of anti-angina doses of nitroglycerin (vascular endothelium-independent dilation function), namely after the sublingual administration of $400 \mu \mathrm{g}$ nitroglycerin after resting. The ultrasonic scanning cycle is $15-60 \mathrm{sec}$ before the cuff discharge.

Determination of related inflammatory factors. The tumor necrosis factor- $\alpha$ (TNF- $\alpha)$, high-sensitivity C-reactive protein (hs-CRP) and nitric oxide (NO) levels were detected via enzyme-linked immunosorbent assay (ELISA). The serum NO content was detected using the Griess reagent staining method; after standardized test, hs-CRP was detected using the LX-20 full-automatic biochemical analyzer manufactured by Beckman Coulter, Inc. (Brea, CA, USA); TNF- $\alpha$ was detected via ELISA using the microplate reader (the kit was from R\&D Systems, Inc., Minneapolis, MN,USA). The reagents were placed in the constant temperature water bath until it reached the room temperature, and the experiment was performed in strict accordance with the instructions of the kit. After reagent preparation and sample loading, the plate was washed with ZM X-988B full-automatic plate washer 3 times; the optical density (OD) value at $450 \mathrm{~nm}$ was detected using the an-thos2010 full-automatic microplate reader (Bio-Rad Laboratories, Inc., Hercules, USA); the standard curve was drawn using the computer software; OD value of the sample $=$ OD value measured - OD value of the blank control; TNF- $\alpha$ concentration of the corresponding sample was checked in the OD value curve of the standard sample.

Incidence of CVDs. The incidence of CVDs within 10 years was summarized and the incidence rates were scored using the Framingham equation, prospective cardiovascular Munster study (PROCAM) and National Cholesterol Education Program Adult Treatment Expert Panel III (NCEP-ATPIII) score. Framingham equation includes the sex, age, DM, hypertension, smoking, total cholesterol and high-density lipoprotein (HDL) cholesterol, and electrocardiogram of left ventricular hypertrophy ( $\mathrm{Y} / \mathrm{N})$; PROCAM is based on the sex, age, blood pressure, smoking, total cholesterol and low-density lipoprotein cholesterol, TG, family history of myocardial infarction, DM and angina; ATPIII score is based on the sex, age, basal blood pressure, DM, smoking, total cholesterol and HDL cholesterol, and family history of early-onset CVDs.

Statistical analysis. Statistical Product and Service Solutions (SPSS) v.19.0 software (SPSS, Inc., Chicago, IL, USA) was used for data processing. Measurement data are presented as (mean \pm standard deviation); Chi-square test was used for the enumeration data, and the t-test was used for the intergroup comparison. Pearson's correlation analysis was performed for the correlation analysis. $\mathrm{P}<0.05$ was considered to indicate a statistically significant difference.

\section{Results}

Comparison of serum TNF- $\alpha$ and hs-CRP between the two groups. The level of hs-CRP and TNF- $\alpha$ in the NAFLD group was significantly increased compared with that in the simple CHD group ( $\mathrm{P}<0.05$; Fig. 1).

Comparison of general conditions and liver function between the two groups. The results of comparisons of BMI and liver function between the two groups showed that the serum TBIL and ALT levels and BMI in the NAFLD group were significantly higher than those in the simple CHD group, and the differences were statistically significant $(\mathrm{P}<0.05$; Fig. 2A-C). There was no statistically significant difference in the serum AST level between the two groups (P>0.05; Fig. 2A-D).

Correlation between liver function (ALT) and vascular endothelial dilation function. The Pearson's correlation analysis 


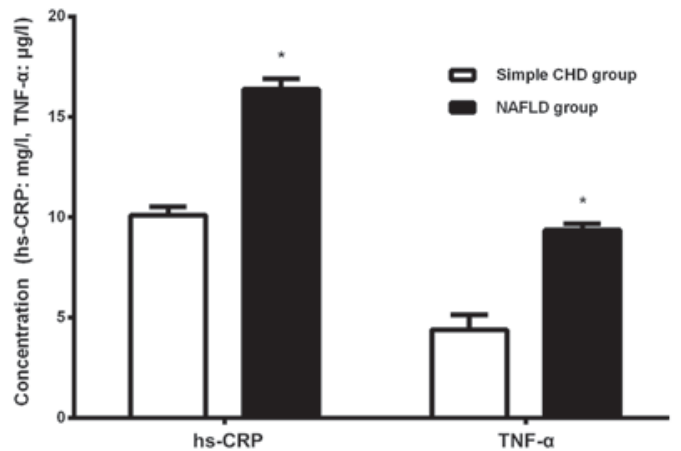

Figure 1. Comparison of serum TNF- $\alpha$ and hs-CRP between the groups. TNF- $\alpha$, tumor necrosis factor- $\alpha$; hs-CRP, high-sensitivity C-reactive protein $\mathrm{CHD}$, coronary heart disease; NAFLD, non-alcoholic fatty liver disease. ${ }^{*} \mathrm{P}<0.05$.

revealed that there was a linearly positive correlation between ALT and brachial artery diameter in the NAFLD group $(\mathrm{r}=0.311, \mathrm{P}<0.05)$; there was a statistically significant difference in the correlation between HOMA-IR and HOMA- $\beta$ indexes and brachial artery diameter in the groups $(r=-0.128, r=0.219$, $\mathrm{P}<0.05)$; there was also a statistically significant difference in the correlation between hs-CRP and brachial artery diameter in the groups $(r=-0.312, \mathrm{P}<0.05)$, but TNF- $\alpha$ had no correlation with brachial artery diameter, and the difference was not statistically significant (r=3.286, P>0.05; Fig. 3A-E).

Comparison of islet function and vascular endothelial dilation function between the two groups. The HOMA-IR and HOMA- $\beta$ indexes in the NAFLD group were obviously decreased compared with those in the simple CHD group, and the differences were statistically significant $(\mathrm{P}<0.01)$, but FBG had no statistically significant difference between the two groups $(\mathrm{P}>0.05$; Table I).

Correlation between serum NO and ALT levels. The Pearson's correlation analysis of serum ALT and NO levels revealed that there was a linearly negative correlation between them ( $r=-0.325, \mathrm{P}<0.05$; Fig. 4).

Comparison of intima-media thickness (IMT), number of carotid plaques and detection rate of carotid plaques between the two groups. There were significant differences in the IMT, number of carotid plaques and detection rate of carotid plaques between the two groups $(\mathrm{P}<0.05$; Table II).

Prognoses of the two groups. The incidence rate of CVDs within 10 years was divided into very low $(<1 \%)$, low $(1-3 \%)$, moderate (4-6\%), high (7-10\%) and very high $(>10 \%)$. The risk of cardiovascular events within 10 years in the NAFLD group was slightly increased compared with that in the simple CHD group. According to the Framingham equation, PROCAM and NCEP-ATPIII score, the differences were statistically significant between the two groups $(\mathrm{P}<0.05$; Table III).

\section{Discussion}

NAFLD is a clinically pathological syndrome characterized by parenchymal liver cells and fat accumulation in the absence of excessive drinking. The main pathological feature of CHD is coronary atherosclerosis, and it mainly occurs in the middle-aged and elderly people, which may be caused by genetic and environmental factors. Abnormal lipid metabolism is often considered to be the main factors of increased blood lipids, and hyperlipidemia is also considered as the common cause of CHD and NAFLD. The clinical diagnosis rate of CHD complicated with NAFLD is very high. Recently it is confirmed that NAFLD is very closely related to the incidence of CHD $(10,11)$. The pathogenesis of NAFLD and CHD is mainly systemic chronic inflammation, gradually enhanced oxidative stress and vascular endothelial dysfunction (12). In addition, several studies have shown that IR refers to the fact that the tissues and organs can not produce normal physiological and biological reactions to a certain amount of insulin, which is the common metabolic defect of many metabolic diseases and considered as an idiopathic feature of NAFLD $(13,14)$, even for subjects without obesity (15). Compared with simple CHD patients, CHD patients complicated with NAFLD will produce IR, cause the release of cytokines and a series of inflammatory reactions, promote the release of serum APN, TNF- $\alpha$, hs-CRP and other factors and increase the mitochondrial oxidative burden of liver cells. A large number of activated oxygen-free radicals bind to the corresponding factors to form peroxides, manifested as coronary atherosclerosis. In addition, CHD patients complicated with NAFLD suffer from vascular endothelial dilation dysfunction, IR and abnormal liver function to some extent, significantly slowing down the blood vessel velocity combined with the common role of inflammatory factors, which is manifested directly as platelet aggregation, thus affecting the vascular endothelial dilation function and prognosis (16).

The results of comparisons of BMI and liver function between the two groups showed that the serum TBIL and ALT levels and BMI in the NAFLD group were significantly higher than those in the simple CHD group, and the differences were statistically significant; there was no statistically significant difference in the serum AST level between the two groups. The Pearson's correlation analysis revealed that there was a linearly positive correlation between ALT and brachial artery diameter in the NAFLD group $(\mathrm{r}=0.311, \mathrm{P}<0.05)$. The HOMA-IR and HOMA- $\beta$ indexes in the NAFLD group were obviously increased compared with those in the simple CHD group, and the differences were statistically significant $(\mathrm{P}<0.01)$; but the FBG had no statistically significant difference between the two groups $(\mathrm{P}>0.05)$. Moreover, the base value of carotid diameter $\left(D_{\mathrm{o}}\right)$ had no statistically significant difference between the two groups $(\mathrm{P}>0.05)$, and it was significantly decreased in the NAFLD group compared with that in the simple CHD group. There were significant differences in the IMT, number of carotid plaques and detection rate of carotid plaques between the two groups $(\mathrm{P}<0.05)$. There was a statistically significant difference in the correlation between HOMA-IR and HOMA- $\beta$ indexes and brachial artery diameter in both groups $(\mathrm{r}=-0.128$, $\mathrm{r}=0.219, \mathrm{P}<0.05)$. There was also a statistically significant difference in the correlation between hs-CRP and brachial artery diameter in the groups $(\mathrm{r}=-0.312, \mathrm{P}<0.05)$, but TNF- $\alpha$ had no correlation with brachial artery diameter, and the difference was not statistically significant $(r=3.286, P>0.05)$. Epidemiological data show that the mortality of CVD is significantly increased in patients diagnosed with NAFLD (17). 
A

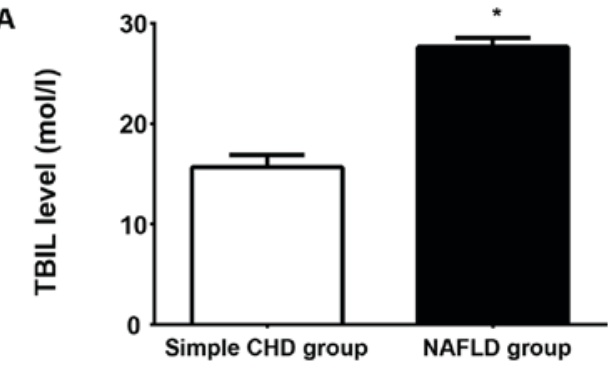

C

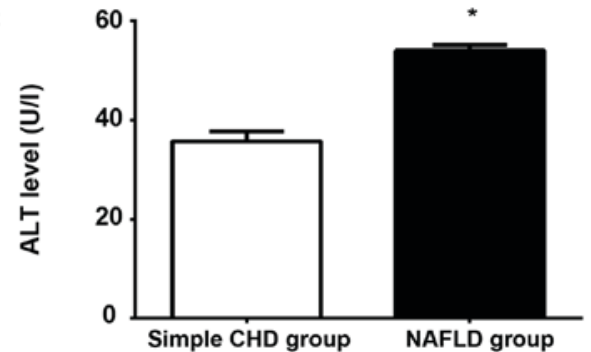

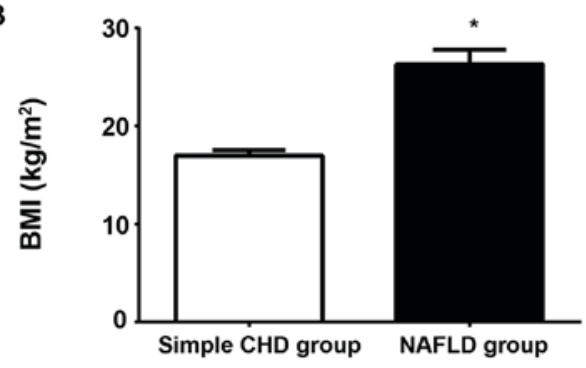

D

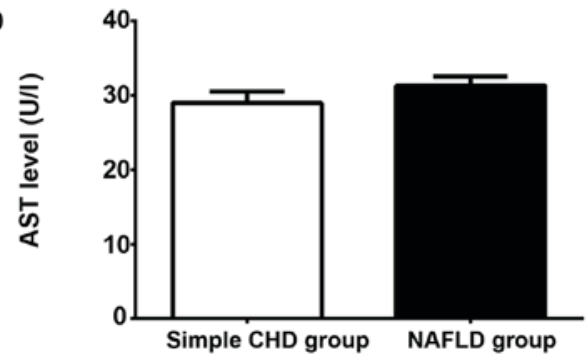

Figure 2. Comparison of general conditions and liver function between the two groups: (A-C) the serum TBIL and ALT levels and BMI in the NAFLD group were significantly higher than those in the simple CHD group, and the differences were statistically significant $\left({ }^{*} \mathrm{P}<0.05\right)$. (D) There was no statistically significant difference in the serum AST level between the two groups $(\mathrm{P}>0.05)$. TBIL, total bilirubin; ALT, alanine aminotransferase; BMI, body mass index; NAFLD, non-alcoholic fatty liver disease; CHD, coronary heart disease; AST, aspartate aminotransferase.

A

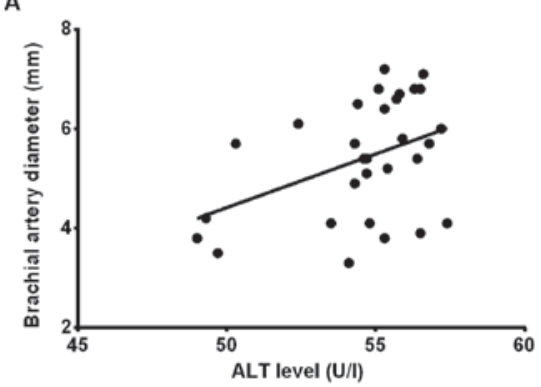

C

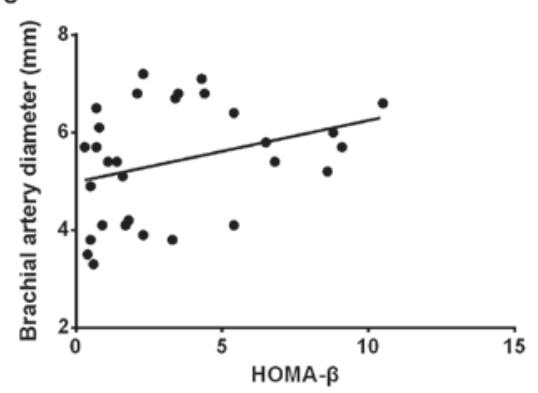

$\mathrm{E}$

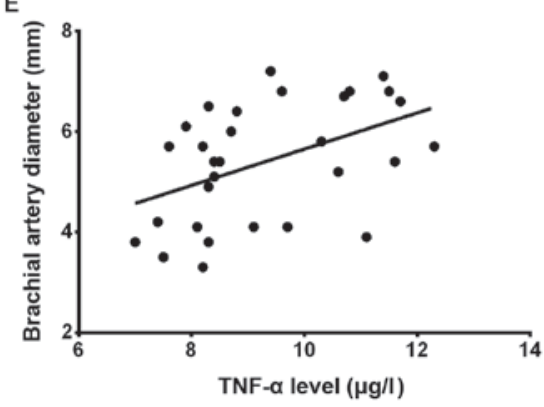

B
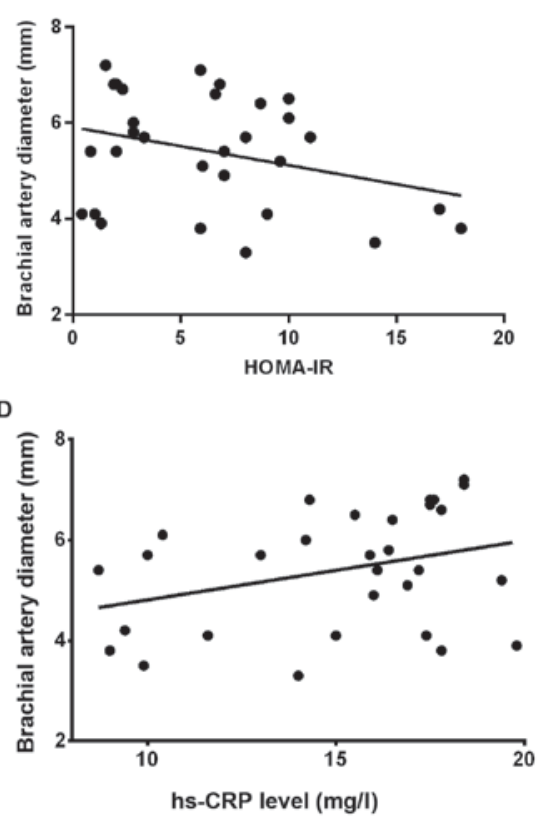

Figure 3. (A) Correlation analysis between serum ALT and brachial artery diameter in the NAFLD group showed that there was a statistically significant difference $(r=0.311, P<0.05)$. (B and $C$ ) There was a statistically significant difference in the correlation between HOMA-IR and HOMA- $\beta$ indexes and brachial artery diameter in both groups $(\mathrm{r}=-0.128, \mathrm{r}=0.219, \mathrm{P}<0.05)$. ( $\mathrm{D}$ and $\mathrm{E})$ There was a statistically significant difference in the correlation between hs-CRP and brachial artery diameter in both groups $(\mathrm{r}=-0.312, \mathrm{P}<0.05)$; TNF- $\alpha$ had no correlation with brachial artery diameter, and the difference was not statistically significant $(\mathrm{r}=3.286, \mathrm{P}>0.05)$. ALT, alanine aminotransferase; NAFLD, non-alcoholic fatty liver disease; HOMA-IR, homeostasis model assessment-insulin resistance; hs-CRP, high-sensitivity C-reactive protein; TNF- $\alpha$, tumor necrosis factor- $\alpha$. 
Table I. Comparison of islet function between the two groups.

\begin{tabular}{lcccc}
\hline Groups & FBG $(\mathrm{mmol} / \mathrm{l})$ & HOMA-IR & HOMA- $\beta$ & FMV (mm) \\
\hline NAFLD group & $7.9 \pm 3.8$ & $4.0 \pm 3.2$ & $26.2 \pm 18.3$ & $3.36 \pm 0.62$ \\
Simple CHD group & $8.3 \pm 3.4$ & $2.6 \pm 3.5$ & $19.8 \pm 10.1$ & $4.27 \pm 0.54$ \\
t & 1.16 & 3.86 & 4.21 & 3.48 \\
P-value & 0.162 & $<0.001$ & 0.001 & 0.062 \\
\hline
\end{tabular}

FBG, fasting blood glucose; HOMA-IR, homeostasis model assessment-insulin resistance.

Table II. Comparisons of IMT, number of carotid plaques and detection rate of carotid plaques between the two groups.

\begin{tabular}{lcccc}
\hline Groups & $\mathrm{n}$ & IMT $(\mathrm{mm})$ & Number of carotid plaques & Detection rate of carotid plaques $(\%)$ \\
\hline NAFLD group & 41 & $1.21 \pm 0.05$ & $21.3 \pm 1.52$ & $72.2 \%$ \\
Simple CHD group & 39 & $0.29 \pm 0.03$ & $1.30 \pm 1.56$ & $55.4 \%$ \\
P-value & & 0.041 & 0.032 & 0.028 \\
\hline
\end{tabular}

IMT, intima-media thickness; NAFLD, non-alcoholic fatty liver disease; CHD, coronary heart disease.

Table III. Comparison of cardiovascular risk between the two groups.

\begin{tabular}{|c|c|c|c|c|c|c|}
\hline \multirow[b]{2}{*}{$\begin{array}{l}\text { Risk of cardiovascular } \\
\text { events within } 10 \text { years }\end{array}$} & \multicolumn{2}{|c|}{ Framingham score } & \multicolumn{2}{|c|}{ PROCAM score } & \multicolumn{2}{|c|}{ ATPIII score } \\
\hline & $\begin{array}{l}\text { NAFLD } \\
\text { group }\end{array}$ & $\begin{array}{l}\text { Simple CHD } \\
\text { group }\end{array}$ & $\begin{array}{l}\text { NAFLD } \\
\text { group }\end{array}$ & $\begin{array}{l}\text { Simple CHD } \\
\text { group }\end{array}$ & $\begin{array}{l}\text { NAFLD } \\
\text { group }\end{array}$ & $\begin{array}{l}\text { Simple CHD } \\
\text { group }\end{array}$ \\
\hline Median, \%(IQR) & $1.2(5.1)$ & $3.7(6.7)$ & $0.5(1.1)$ & $0.8(2.2)$ & $<1.0(2.5)$ & $1.5(4.0)$ \\
\hline$<1 \%$ & 43 & 13 & 57 & 37 & 54 & 29 \\
\hline $1-3 \%$ & 21 & 42 & 36 & 33 & 25 & 40 \\
\hline $4-6 \%$ & 18 & 12 & 013 & 14 & 11 & \\
\hline $7-10 \%$ & 7 & 23 & 74 & 7 & 10 & \\
\hline$>10 \%$ & 11 & 10 & 013 & 0 & 10 & \\
\hline P-value & 0.017 & 0.045 & 0.129 & & & \\
\hline
\end{tabular}

IQR and percentage of each risk are used for data in both groups. IQR, interquartile range; NAFLD, non-alcoholic fatty liver disease; CHD, coronary heart disease; PROCAM, prospective cardiovascular Munster; ATPIII, Adult Treatment Expert Panel III.

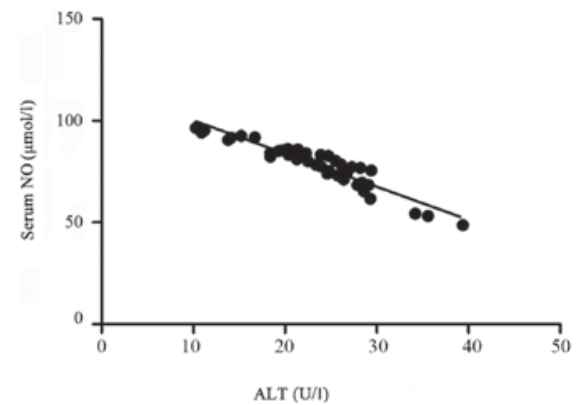

Figure 4. Correlation between serum NO and ALT levels. NO, nitric oxide; ALT, alanine aminotransferase.

Similarly, a recent study showed that compared with the control group, the average IMT and plaque detection rate are higher in NAFLD patients with carotid atherosclerotic, which is consistent with this study. The risk of cardiovascular events within 10 years in the NAFLD group was slightly increased compared with that in the simple CHD group. According to the Framingham equation, PROCAM and NCEP-ATPIII score, the differences were statistically significant between the two groups $(\mathrm{P}<0.05)$. The results of this study showed that the level of hs-CRP and TNF- $\alpha$ in the NAFLD group was significantly increased compared with that in the simple CHD group $(\mathrm{P}<0.05)$, suggesting that TNF- $\alpha$ and hs-CRP are of great significance in the occurrence and development of CHD complicated with NAFLD. Lomonaco et al (18) found that the serum TNF- $\alpha$ level is an independent predictor for the acute myocardial infarction and death of CHD patients. Some scholars have proposed (19) that TNF- $\alpha$ is related to the formation of atherosclerosis in CHD patients, which mainly 
acts on the endothelial cells to produce the platelet-derived growth factor and promote the smooth muscle cell migration and proliferation, ultimately leading to the endothelial cell dysfunction and intima thickening, so it is involved in the occurrence and development of atherosclerosis.

Pearson's correlation analysis of serum ALT and NO levels showed that there was a linearly positive correlation between them $(\mathrm{r}=0.325, \mathrm{P}<0.05)$. The serum NO is considered to be an important index of endothelial function; its correlation with the vascular endothelial dilatation function is not clearly reported yet, but the NO content is decreased with the increase of serum ALT level in NAFLD patients (20). Therefore, the changes in liver function indexes, IR and related inflammatory factors in CHD patients complicated with NAFLD significantly affect the vascular endothelial dilation function, which also have some effects on the occurrence of CVDs.

\section{Acknowledgements}

Not applicable.

\section{Funding}

No funding was received.

\section{Availability of data and materials}

The datasets used and/or analyzed during the current study are available from the corresponding author on reasonable request.

\section{Authors' contributions}

BW wrote the manuscript. BW and FL performed ELISA. JG and CW worked on IR assessment. DX and CL were responsible for statistical analysis. All authors read and approved the final manuscript.

\section{Ethics approval and consent to participate}

The study was approved by the Ethics Committee of Jinan Central Hospital (Jinan, China) and signed informed consents were obtained by the patients or guardians.

\section{Patient consent for publication}

Not applicable.

\section{Competing interests}

The authors declare that they have no competing interests.

\section{References}

1. Szczepaniak LS, Babcock EE, Schick F, Dobbins RL, Garg A, Burns DK, McGarry JD and Stein DT: Measurement of intracellular triglyceride stores by $\mathrm{H}$ spectroscopy: validation in vivo. Am J Physiol 276: E977-E989, 1999.

2. Browning JD, Szczepaniak LS, Dobbins R, Nuremberg P, Horton JD, Cohen JC, Grundy SM and Hobbs HH: Prevalence of hepatic steatosis in an urban population in the United States: Impact of ethnicity. Hepatology 40: 1387-1395, 2004.

3. Day CP: Pathogenesis of steatohepatitis. Best Pract Res Clin Gastroenterol 16: 663-678, 2002.
4. Chalasani N, Younossi Z, Lavine JE, Diehl AM, Brunt EM, Cusi K, Charlton M and Sanyal AJ; American Gastroenterological Association; American Association for the Study of Liver Diseases; American College of Gastroenterologyh: The diagnosis and management of non-alcoholic fatty liver disease: practice guideline by the American Gastroenterological Association, American Association for the Study of Liver Diseases, and American College of Gastroenterology. Gastroenterology 142: 1592-1609, 2012.

5. Fracanzani AL, Valenti L, Bugianesi E, Andreoletti M, Colli A, Vanni E, Bertelli C, Fatta E, Bignamini D, Marchesini G, et al: Risk of severe liver disease in nonalcoholic fatty liver disease with normal aminotransferase levels: a role for insulin resistance and diabetes. Hepatology 48: 792-798, 2008.

6. Kotronen A, Juurinen L, Hakkarainen A, Westerbacka J, Cornér A, Bergholm R and Yki-Järvinen H: Liver fat is increased in type 2 diabetic patients and underestimated by serum alanine aminotransferase compared with equally obese nondiabetic subjects. Diabetes Care 31: 165-169, 2008.

7. Gastaldelli A, Cusi K, Pettiti M, Hardies J, Miyazaki Y, Berria R, Buzzigoli E, Sironi AM, Cersosimo E, Ferrannini E, et al: Relationship between hepatic/visceral fat and hepatic insulin resistance in nondiabetic and type 2 diabetic subjects. Gastroenterology 133: 496-506, 2007.

8. Bellentani S, Saccoccio G, Masutti F, Crocè LS, Brandi G, Sasso F, Cristanini $\mathrm{G}$ and Tiribelli C: Prevalence of and risk factors for hepatic steatosis in Northern Italy. Ann Intern Med 132: 112-117, 2000.

9. Bhatia LS, Curzen NP, Calder PC and Byrne CD: Non-alcoholic fatty liver disease: a new and important cardiovascular risk factor? Eur Heart J 33: 1190-1200, 2012.

10. Sunny NE, Parks EJ, Browning JD and Burgess SC: Excessive hepatic mitochondrial TCA cycle and gluconeogenesis in humans with nonalcoholic fatty liver disease. Cell Metab 14: 804-810, 2011.

11. Souza MR, Diniz MF, Medeiros-Filho JE and Araújo MS: Metabolic syndrome and risk factors for non-alcoholic fatty liver disease. Arq Gastroenterol 49: 89-96, 2012.

12. Chapman MJ, Ginsberg HN, Amarenco P, Andreotti F, Borén J, Catapano AL, Descamps OS, Fisher E, Kovanen PT, Kuivenhoven JA, et al; European Atherosclerosis Society Consensus Panel: Triglyceride-rich lipoproteins and high-density lipoprotein cholesterol in patients at high risk of cardiovascular disease: evidence and guidance for management. Eur Heart J 32: 1345-1361, 2011.

13. Sanyal AJ, Campbell-Sargent C, Mirshahi F, Rizzo WB, Contos MJ, Sterling RK, Luketic VA, Shiffman ML and Clore JN: Nonalcoholic steatohepatitis: Association of insulin resistance and mitochondrial abnormalities. Gastroenterology 120 : 1183-1192, 2001.

14. Yki-Järvinen H: Liver fat in the pathogenesis of insulin resistance and type 2 diabetes. Dig Dis 28: 203-209, 2010.

15. Fabbrini E, Magkos F, Mohammed BS, Pietka T, Abumrad NA, Patterson BW, Okunade A and Klein S: Intrahepatic fat, not visceral fat, is linked with metabolic complications of obesity. Proc Natl Acad Sci USA 106: 15430-15435, 2009.

16. Bugianesi E, Gastaldelli A, Vanni E, Gambino R, Cassader M, Baldi S, Ponti V, Pagano G, Ferrannini E and Rizzetto M: Insulin resistance in non-diabetic patients with non-alcoholic fatty liver disease: sites and mechanisms. Diabetologia 48: 634-642, 2005.

17. Wald D, Teucher B, Dinkel J, Kaaks R, Delorme S, Boeing H, Seidensaal K, Meinzer HP and Heimann T: Automatic quantification of subcutaneous and visceral adipose tissue from whole-body magnetic resonance images suitable for large cohort studies. J Magn Reson Imaging 36: 1421-1434, 2012.

18. Lomonaco R, Ortiz-Lopez C, Orsak B, Webb A, Hardies J, Darland C, Finch J, Gastaldelli A, Harrison S, Tio F, et al: Effect of adipose tissue insulin resistance on metabolic parameters and liver histology in obese patients with nonalcoholic fatty liver disease. Hepatology 55: 1389-1397, 2012.

19. Machado MV, Ferreira DM, Castro RE, Silvestre AR, Evangelista T, Coutinho J, Carepa F, Costa A, Rodrigues CM and Cortez-Pinto $\mathrm{H}$ : Liver and muscle in morbid obesity: the interplay of fatty liver and insulin resistance. PLoS One 7: e31738, 2012.

20. Gastaldelli A: Role of beta-cell dysfunction, ectopic fat accumulation and insulin resistance in the pathogenesis of type 2 diabetes mellitus. Diabetes Res Clin Pract 93 (Suppl 1): S60-S65, 2011. 